\title{
Area inside a Circle: Intuitive and Rigorous Proofs
}

\author{
Vali Siadat \\ Department of Mathematics, Richard J. Daley College, Chicago, IL, USA \\ Email:vsiadat@ccc.edu
}

How to cite this paper: Siadat, V. (2017) Area inside a Circle: Intuitive and Rigorous Proofs. American Journal of Computational Mathematics, 7, 102-108.

https://doi.org/10.4236/ajcm.2017.71009

Received: February 10, 2017

Accepted: March 28, 2017

Published: March 31, 2017

Copyright (C) 2017 by author and Scientific Research Publishing Inc. This work is licensed under the Creative Commons Attribution International License (CC BY 4.0).

http://creativecommons.org/licenses/by/4.0/

\begin{abstract}
In this article I conduct a short review of the proofs of the area inside a circle. These include intuitive as well as rigorous analytic proofs. This discussion is important not just from mathematical view point but also because pedagogically the calculus books still use circular reasoning today to prove the area inside a circle (also that of an ellipse) on this important historical topic, first illustrated by Archimedes. I offer an innovative approach through the introduction of a theorem, which will lead to proving the area inside a circle avoiding circular argumentation.
\end{abstract}

\section{Keywords}

Area, Circle, Ellipse, Circular Reasoning, Intuitive Proof, Rigorous Proof

\section{Introduction}

Why do we discuss the area inside a circle again? Why we shouldn't confound the notions of intuition and rigor? Do calculus books, even today, still resort to circular reasoning? This paper is an attempt to elucidate these questions by walking the reader through the path of intuitive to solid analytical reasoning, pointing out the gaps that often occur on the proof of this ancient and well known problem, which is first illustrated by Archimedes. The motivation behind writing of this piece was to engage the reader in further thinking about mathematical proofs and the level of rigor at which they are presented.

In the following we present a brief review of the proofs of area inside a circle. A typical rigorous proof requires knowledge of integral calculus, seeing example [1]. But even in these proofs presented by calculus books, seeing the example [2], the authors resort to circular reasoning. To prove the area inside a circle, they set up the integral $\int_{0}^{1} \sqrt{1-x^{2}} \mathrm{~d} x$ followed by trigonometric substitution which requires knowing that the derivative of $\sin \theta$ is $\cos \theta$. But this latter fact requires 
proving that $\lim _{\theta \rightarrow 0} \frac{\sin \theta}{\theta}=1$. For this proof, they resort to a geometric argument, bounding the area of a sector of a unit circle between the areas of two triangles and showing that $\sin \theta<\theta<\tan \theta$. They then apply the Squeeze Theorem. But for computation of the sector's area, they resort to a standard formula, $A=\frac{1}{2} \theta$, which is based on knowing the area of a circle. So they prove the area by assuming the area. This is obviously circular argumentation! For an excellent critique of this method we can see [3].

There are also a number of intuitive proofs intended to provide an insight to the derivation of the area with just the knowledge of geometry and limits. In this short piece we begin by proving a preliminary result showing that $\lim _{\theta \rightarrow 0} \frac{\sin \theta}{\theta}=1$, without a priori assuming the area of a sector. This limit is central to the proof of the derivatives of trigonometric functions. We note that aside from the aforementioned limit, the function $\frac{\sin \theta}{\theta}$ itself plays an important role not only in mathematics but also in other fields of science such as physics and engineering.

\section{Proofs}

Consider a circle of radius 1 which is centered at the origin, as shown in Figure 1; see [4].

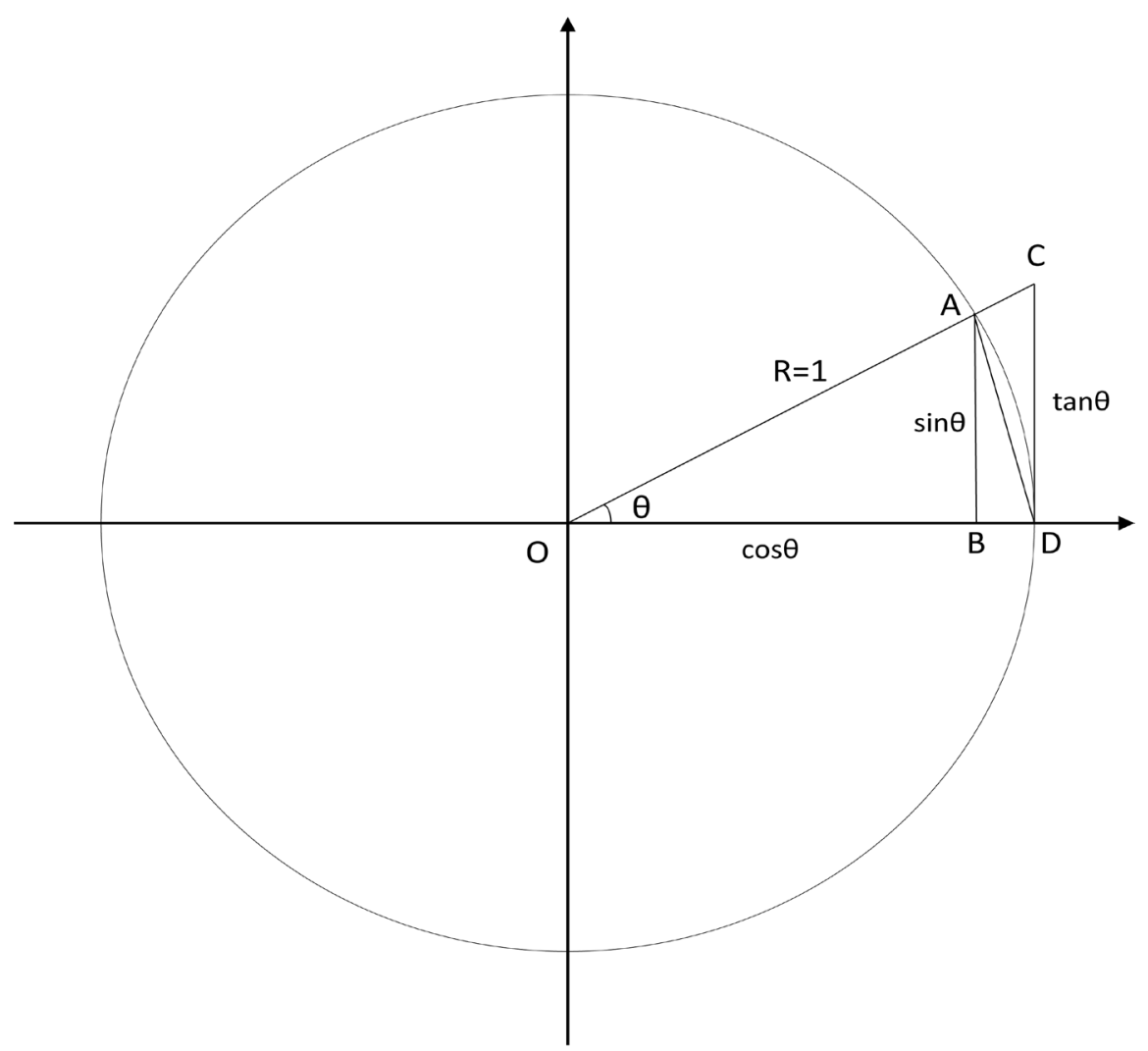

Figure 1. A unit circle in standard position. 
Theorem 1. Let $0<\theta<\frac{\pi}{2}$ be an angle measured in radians. Then,

$$
\lim _{\theta \rightarrow 0} \frac{\sin \theta}{\theta}=1
$$

Proof. Since the magnitude of $\theta$ equals the length of the arc it subtends and since $\sin \theta<\overline{A D}$, we have that $\sin \theta<\theta$, or $1<\frac{\theta}{\sin \theta}$. This establishes a lower bound. To establish the upper bound, we can show that $\theta<\sin \theta+\overline{B D}$. This can be proved by the standard method of estimating an arc length of a rectifiable curve by the linear approximation of the lengths of the chords it subtends through partitioning. The result follows by applying the triangle inequality in each partition in [3]. Noting that $\sin \theta<\tan \theta$ we get $\theta<\tan \theta+\overline{B D}$ But $\overline{B D}=1-\cos \theta$. So it follows that,

$$
\frac{\theta}{\sin \theta}<\frac{1}{\cos \theta}+\frac{1-\cos \theta}{\sin \theta}
$$

and

$$
\frac{1-\cos \theta}{\sin \theta}=\sqrt{\frac{(1-\cos \theta)^{2}}{1-\cos ^{2} \theta}}=\sqrt{\frac{1-\cos \theta}{1+\cos \theta}} .
$$

Combining this result with the previous lower bound gives,

$$
1<\frac{\theta}{\sin \theta}<\frac{1}{\cos \theta}+\sqrt{\frac{1-\cos \theta}{1+\cos \theta}} .
$$

Letting $\theta \rightarrow 0$ in the last expression completes the upper bound, resulting in $1 \leq \frac{\theta}{\sin \theta} \leq 1$. Finally, applying the Squeeze Theorem we get,

$$
\lim _{\theta \rightarrow 0} \frac{\sin \theta}{\theta}=1
$$

An interesting question related to the foregoing bounding of the angle $\theta$ is that if we define the derivatives of trigonometric functions of $\theta$ analytically (i.e., by infinite series of complex numbers or solutions of differential equations), can we arrive at the bounding of the angle? The next theorem follows.

Theorem 2. If $(\sin \theta)^{\prime}=\cos \theta$, and $(\tan \theta)^{\prime}=\sec ^{2} \theta$, then

$$
\sin \theta \leq \theta \leq \tan \theta .
$$

Proof. Let $f(\theta)=\sin (\theta)-\theta$ than $f(0)=0$ and $f^{\prime}(\theta)=\cos \theta-1 \leq 0$, giving $f^{\prime}(\theta)<0$ for $\theta>0$.

Hence,

$$
f(\theta)=\int_{0}^{\theta} f^{\prime}(t) \mathrm{d} t<0 .
$$

Therefore, $\sin \theta<\theta$. This gives a lower bound for $\theta$.

To find an upper bound for $\theta$, let $g(\theta)=\tan \theta-\theta$.

$g(0)=0$ and $g^{\prime}(\theta)=\sec ^{2} \theta-1 \geq 0$, giving $g^{\prime}(\theta)>0$ for $\theta>0$.

Hence,

$$
g(\theta)=\int_{0}^{\theta} g^{\prime}(t) \mathrm{d} t>0
$$


Therefore, $\theta<\tan \theta$. This gives an upper bound for $\theta$. Combining the above results we get $\sin \theta \leq \theta \leq \tan \theta$.

Theorem 3. Area inside a circle of radius $R$ is $\pi R^{2}$.

Proof. Consider a circle of radius $R$ centered at the origin in Figure 2.

Partition the circle into $n$ equal slices and consider a slice with central angle $\frac{2 \pi}{n}$ radians. We know that the area of a triangle is one-half times the product of two of its sides times the sine of the angle between the two sides. So the area of the triangle subtended by the central angle $\frac{2 \pi}{n}$ becomes $A=\frac{1}{2} R^{2} \sin \left(\frac{2 \pi}{n}\right)$. Because there are $n$ inscribed triangles in the circle, the total area of all these triangles would be $A_{\text {total }}=n \frac{1}{2} R^{2} \sin \left(\frac{2 \pi}{n}\right)$. As we increase the number of slices by increasing $n$, the sum of the areas of the inscribed triangles get closer to the area of the circle. To get the area of the circle, we need to find the limit of $A_{\text {total }}$, as $n \rightarrow \infty$. So, using (1), and since $\frac{2 \pi}{n} \rightarrow 0$, as $n \rightarrow \infty$, the area of the circle becomes:

$$
A_{\text {circle }}=\lim _{n \rightarrow \infty} n \frac{1}{2} R^{2} \sin \left(\frac{2 \pi}{n}\right)=\pi R^{2}\left(\lim _{n \rightarrow \infty} \frac{\sin \frac{2 \pi}{n}}{\frac{2 \pi}{n}}\right)=\pi R^{2} .
$$

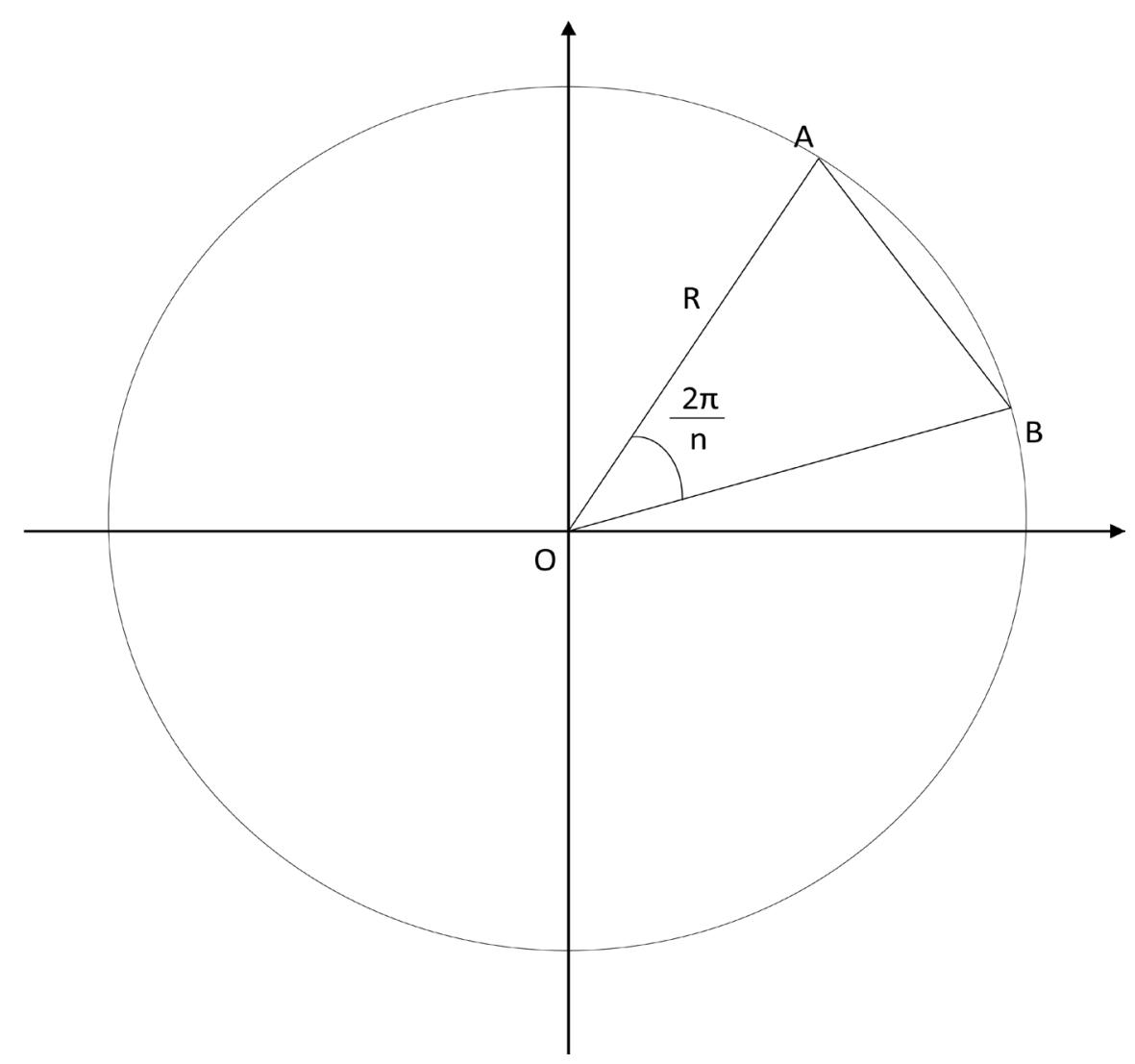

Figure 2. A circle of radius $R$ with $n$ equal slices. 
Hence, $A_{\text {circle }}=\pi R^{2}$.

An intuitive and interesting method of proving the area inside a circle which requires area stretching ${ }^{1}$ and mapping from an annulus to a trapezoid is discussed in [5]. The subtle point in this method, as expressed in [5], is that it assumes as evident the area preservation from a circular to a simply connected region. For a discussion of transformation of different regions by using complex variable method, we can see [6].

There are many other intuitive approaches also, some of which involve slicing or opening up a circle. Below we offer a simple intuitive proof which is not based on area stretching, but assumes area preservation under mappings. Consider two concentric circles with radii $r$ and $R$ and corresponding areas $A_{r}$ and $A_{R}$. Cut the annulus open in the shape of a right angle trapezoid $A B C D$ as in Figure 3.

We can see that the area of the annulus equals the area of the trapezoid. So, $A_{\text {annulus }}=A_{\text {trapezoid }}$, or

$$
A_{R}-A_{r}=\frac{1}{2}(2 \pi R+2 \pi r)(R-r)=\pi R^{2}-\pi r^{2} .
$$

We can choose $r>0$ as small as we please and so, in particular, if we let $r$ approach 0 , the area of the inner circle approaches 0 and we get,

$A_{R}-0=\pi R^{2}-0$. Thus $A_{R}=\pi R^{2}$. Note that shrinking $r$ to 0 , shrinks the trapezoid to the right triangle $A B C$, whose area is

$\frac{1}{2}(2 \pi R)(R-0)=\pi R^{2}=A_{R}$. In the following we present an analytic proof of the area inside a circle using area stretching, which does not assume area preserving mapping of regions.

Theorem 4. Area inside a circle of radius $r$ is $\pi r^{2}$.

Proof. Consider a circle of radius $r$ centered at the origin and partition it into $n$ equal sectors, each having central angle $\frac{2 \pi}{n}$, and the corresponding arc length $\frac{2 \pi}{n} r$. Assume the area of a sector is $c_{n}$. If we stretch the radius $r$ by a factor of $k>1$, we create a circle with radius $R=k r$. So, the corresponding streched sector will have an arc length equal to $\frac{2 \pi}{n} k r$ and the its area

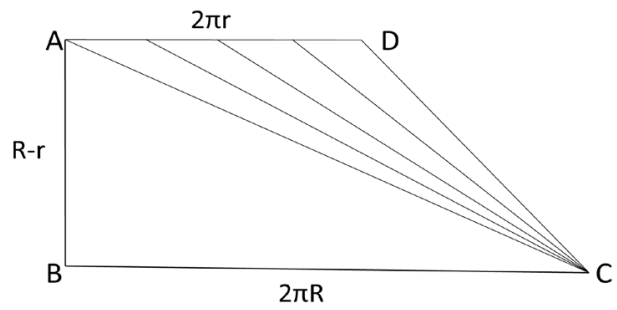

Figure 3. An annulus of a circle cut open in the shape of a trapezoid.

\footnotetext{
${ }^{1}$ Area stretching is a result from geometry stating that if we stretch a region in the coordinate plane vertically by a factor of $k>0$ and horizontally by a factor of $l>0$, then its area will stretch by the factor $k l$.
} 
will be increased by a factor of $k^{2}$ to $k^{2} c_{n}$, seeing in Figure 4. Now the area between the two sectors is $A_{\text {betwensectors }}=k^{2} c_{n}-c_{n}=c_{n}\left[k^{2}-1\right]$ which is approximately equal to the area of the trapezoid $A B C D$ in Figure 4.

If we connect the center $O$ to the point $F$ which is the midpoint of $\overline{B C}$, the triangles $\triangle O B F$ and $\triangle O C F$ become right angle congruent triangles with right angles at the point $F$. As a result, central angles $\angle A O E$ and $\angle D O E$ will each equal $\frac{\pi}{n}$. Obviously triangles $\triangle O A E$ and $\triangle O D E$ are also congruent having right angles at the point $E$. To calculate the area of the trapezoid, we note that its larger base has length $\overline{B C}=2 R \sin \frac{\pi}{n}$, and its smaller base has length $\overline{A D}=2 r \sin \frac{\pi}{n}$. The height of the trapezoid is:

$$
\overline{E F}=R \cos \frac{\pi}{n}-r \cos \frac{\pi}{n}
$$

Therefore the area of the trapezoid becomes:

$$
\begin{aligned}
A_{\text {trapezoid }} & =\frac{1}{2}\left[2 R \sin \frac{\pi}{n}+2 r \sin \frac{\pi}{n}\right] \cdot\left[R \cos \frac{\pi}{n}-r \cos \frac{\pi}{n}\right] \\
& =\sin \frac{\pi}{n} \cos \frac{\pi}{n}\left[R^{2}-r^{2}\right] \\
& =\sin \frac{\pi}{n} \cos \frac{\pi}{n} \cdot r^{2}\left[k^{2}-1\right] .
\end{aligned}
$$

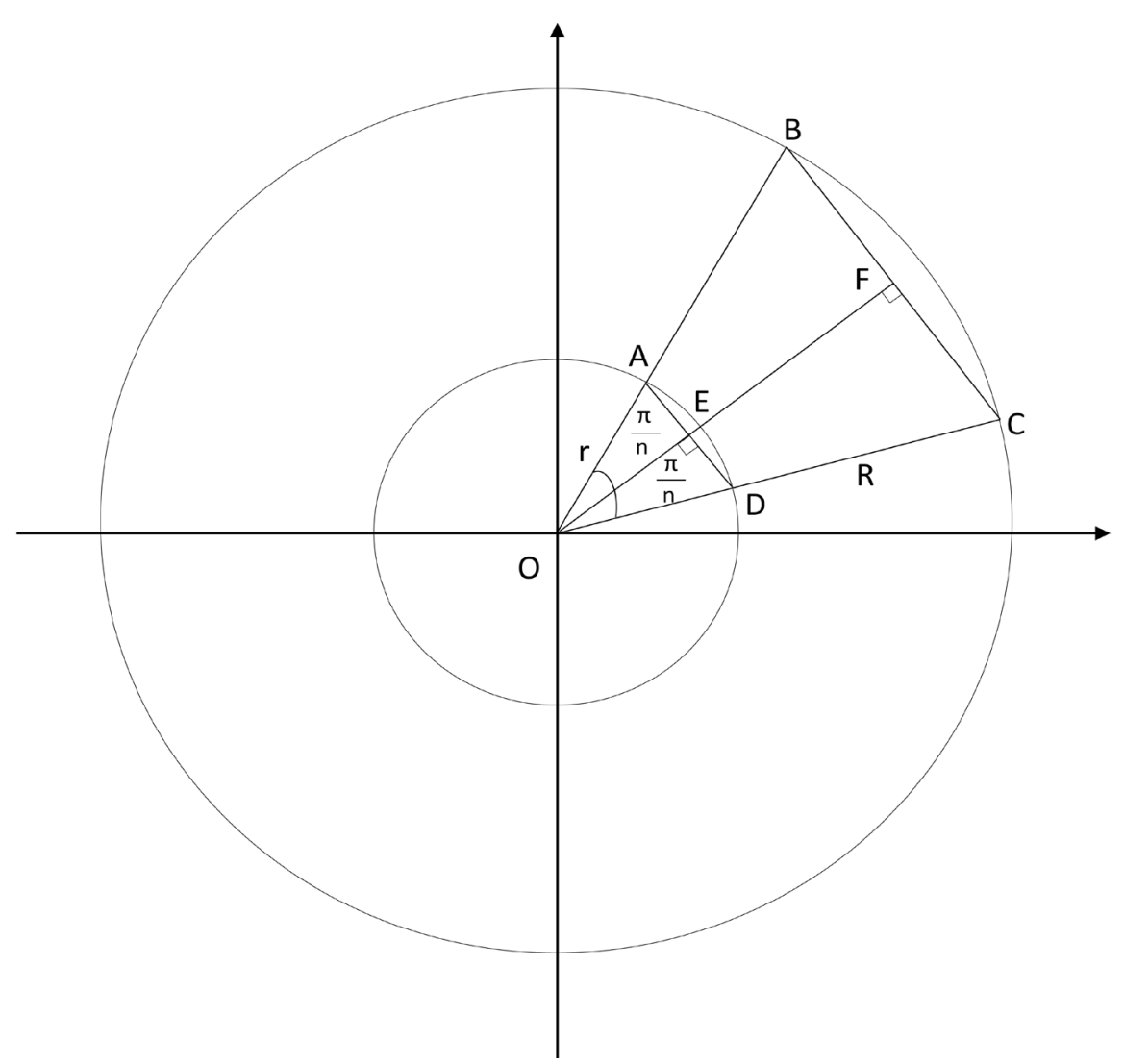

Figure 4. A sector of a circle stretched by a factor $k>1$ to an enhanced sector. 
Setting $A_{\text {betweensectors }} \approx A_{\text {trapezoid }}$, gives,

$$
c_{n}\left[k^{2}-1\right] \approx \sin \frac{\pi}{n} \cos \frac{\pi}{n} \cdot r^{2}\left[k^{2}-1\right],
$$

or $c_{n} \approx \sin \frac{\pi}{n} \cos \frac{\pi}{n} \cdot r^{2}$. This approximation can be improved by increasing $n$. Now, multiplying both sides of the above by $n$ gives:

$$
n c_{n} \approx n \sin \frac{\pi}{n} \cos \frac{\pi}{n} \cdot r^{2}
$$

Since there are exactly $n$ identical sectors in the circle of radius $r$, its area becomes $c=n c_{n}$. Therefore, $c \approx n \sin \frac{\pi}{n} \cos \frac{\pi}{n} \cdot r^{2}$. Now, taking the limit of both sides as $n \rightarrow \infty$, and applying our earlier result (1) and the fact that $\cos \theta \rightarrow 1$, as $\theta \rightarrow 0$, we get:

$$
\begin{aligned}
c & =\lim _{n \rightarrow \infty} n \sin \frac{\pi}{n} \cos \frac{\pi}{n} \cdot r^{2} \\
& =\lim _{n \rightarrow \infty} \pi \cdot \frac{\sin \frac{\pi}{n}}{\frac{\pi}{n}} \cos \frac{\pi}{n} \cdot r^{2} \\
& =\pi r^{2}\left(\lim _{n \rightarrow \infty} \frac{\sin \frac{\pi}{n}}{\frac{\pi}{n}}\right) \cdot\left(\lim _{n \rightarrow \infty} \cos \frac{\pi}{n}\right) \\
& =\pi r^{2}
\end{aligned}
$$

Hence, $c=\pi r^{2}$.

\section{Acknowledgements}

I wish to thank Professor Cyrill Oseledets of Richard J. Daley College for his review of the initial manuscript and making helpful suggestions to improve it.

\section{References}

[1] Larson, R.E. and Bruce, H. (2014) Calculus. 10th Edition, Cengage Learning, Brooks/Cole, Boston, 533-541.

[2] Briggs, W., Cochran, L. and Gillett, B. (2015) Cochran, Lyle. In: Gillett, B., Ed., Calculus, 2nd Edition, Pearson Education, New York, 527-536.

[3] Krantz, S. (1992) On the Area Inside a Circle. Missouri Journal of Mathematics, 4, 2-8.

[4] Siadat, M.V. (2002) Axial View of Trigonometric Functions. Mathematics Magazine, 75, 396-397.

[5] Axler, S. (2013) Precalculus (A Prelude to Calculus). 2nd Edition, John Wiley \& Sons, Inc., Hoboken, 617-628.

[6] Brown, J.W. and Churchill, R.V. (2004) Complex Variables and Applications. 7th Edition, McGraw-Hill Higher Education, Boston, 441-449. 
Submit or recommend next manuscript to SCIRP and we will provide best service for you:

Accepting pre-submission inquiries through Email, Facebook, LinkedIn, Twitter, etc. A wide selection of journals (inclusive of 9 subjects, more than 200 journals)

Providing 24-hour high-quality service

User-friendly online submission system

Fair and swift peer-review system

Efficient typesetting and proofreading procedure

Display of the result of downloads and visits, as well as the number of cited articles Maximum dissemination of your research work

Submit your manuscript at: http://papersubmission.scirp.org/

Or contact ajcm@scirp.org 